\title{
Estado nutricional y nivel de hemoglobina según el tipo de lactancia en el lactante de 6 meses
}

\section{Nutritional status and hemoglobin level according to the type of breastfeeding in the 6-month-old infant}

\author{
Esperanza Luz Buitron Martel « 1,a
}

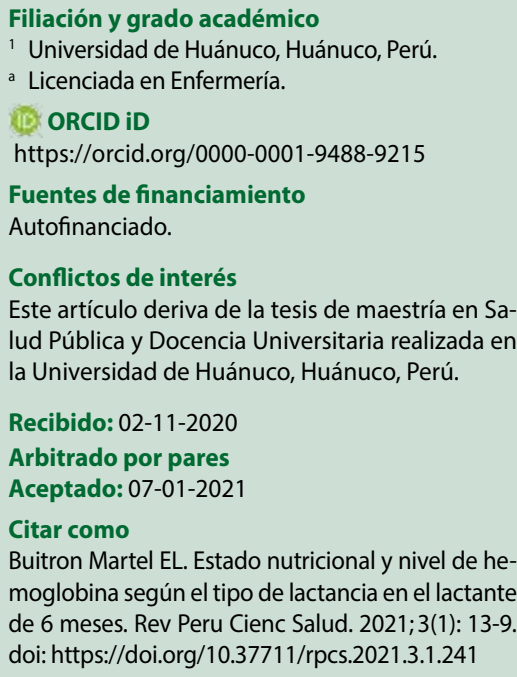

Recibido: 02-11-2020

Arbitrado por pares

Aceptado: 07-01-2021

Citar como

Buitron Martel EL. Estado nutricional y nivel de hemoglobina según el tipo de lactancia en el lactante de 6 meses. Rev Peru Cienc Salud. 2021;3(1): 13-9. doi: https://doi.org/10.37711/rpcs.2021.3.1.241

\section{RESUMEN}

Objetivo. Determinar la relación entre el estado nutricional y el nivel de hemoglobina; el tipo de lactancia y el estado nutricional; el tipo de lactancia y el nivel de hemoglobina del lactante de 6 meses que acudieron al control CRED del Centro de Salud Perú-Corea, Huánuco, 2019. Métodos. Fue un estudio con enfoque cuantitativo, de tipo retrospectivo, analítico y transversal. La población muestral fueron 564 historias clínicas de lactantes de 6 meses. La comprobación de hipótesis fue mediante la prueba chi cuadrado de independencia, considerando un $p \leq 0,05$. Resultados. Evaluando el tipo de lactancia que consumen los lactantes de 6 meses, se evidenció que el 65,6\% (370) continuaron con lactancia materna exclusiva, frente a un $25,7 \%$ (145) con lactancia mixta. El estado nutricional fue normal en mayor proporción [99,1 \% (559)]. El nivel de hemoglobina también, fue mayoritariamente normal [98,9\% (558)]. Se halló relación entre el estado nutricional y el nivel de hemoglobina $\left[\left(x^{2}=488,13 ; p=0,000\right)\right]$, la lactancia materna exclusiva y el estado nutricional normal $\left[\left(x^{2}=25,55 ; p=0,006\right)\right]$ y lactancia materna exclusiva y un nivel normal de hemoglobina $\left[\left(x^{2}=30,71 ; p=0,000\right)\right]$. Conclusiones. Existe relación entre el estado nutricional normal y el nivel normal de la hemoglobina, la lactancia materna exclusiva y el estado nutricional normal; asimismo, se halló relación entre la lactancia materna exclusiva y el nivel normal de hemoglobina de los lactantes.

Palabras clave: estado nutricional; hemoglobina; lactancia materna exclusiva; lactancia materna mixta; lactancia artificial; anemia (Fuente: DeCS-BIREME).

\section{ABSTRACT}

Objective. To determine the relationship between nutritional status and hemoglobin level; type of breastfeeding and nutritional status; type of breastfeeding and hemoglobin level of the 6-month-old infant who attended the CRED control of the Centro de Salud Perú-Corea, Huánuco, 2019. Methods. It was a quantitative, retrospective, analytical and cross-sectional study. The sample population was 564 clinical histories of 6-month-old infants. The hypothesis test was based on the chi square test of independence, considering a $p 0,05$. Results. Evaluating the type of breastfeeding consumed by the 6-month-old infants, it was found that $65.6 \%$ (370) continued with exclusive breastfeeding, compared to $25.7 \%$ (145) with mixed breastfeeding. The nutritional status was normal in a higher proportion [99.1\% (559)]. The nutritional status was normal in a higher proportion [99.1\% (559)]. The hemoglobin level was also mostly normal [98.9 \% (558)]. A relationship was found between nutritional status and hemoglobin level $\left[\left(x^{2}=488.13\right.\right.$; $p=0.000)]$, exclusive breastfeeding and normal nutritional status $\left[\left(x^{2}=\right.\right.$ $25.55 ; p=0.006)]$ and exclusive breastfeeding and normal hemoglobin level $\left[\left(x^{2}=30.71 ; p=0.000\right)\right]$. Conclusions. There is a relationship between normal nutritional status and normal hemoglobin level, exclusive breastfeeding and normal nutritional status; likewise, a relationship was found between exclusive breastfeeding and normal hemoglobin level in infants.

Keywords: nutritional status; hemoglobin; exclusive breastfeeding; mixed breastfeeding; artificial breastfeeding; anemia (Source: MeSH - NLM). 


\section{INTRODUCCIÓN}

Según la Organización Mundial de la Salud (OMS), para asegurar que los niños alcancen un apropiado crecimiento físico y desarrollo definido es necesaria una adecuada nutrición desde los primeros meses de vida. En este contexto, el tipo de lactancia, sobre todo la lactancia materna exclusiva, es de vital importancia. La leche humana es un alimento de alto valor nutricional en el patrón de ganancia de peso y talla durante el primer año de vida ${ }^{(1)}$. Tal como señalan Barriuso y Sánchez ${ }^{(2)}$, la composición nutricional de la leche materna es de un valor nutricional incomparable; pero no es fija, sino que se adecua idóneamente al momento de la mamada, al momento del día y a la edad gestacional y posconcepcional del niño.

Es así que el tipo de lactancia durante el primer año de vida, es clave en los indicadores del crecimiento infantil ${ }^{(3,4)}$. Es posible brindar alimentos complementarios, seguros, y apropiados, a partir de los seis meses de edad del niño hasta los 2 años o más.

Pese a las evidencias científicas del valor nutricional de la lactancia materna exclusiva, su cumplimiento es de nivel bajo. Según Choque ${ }^{(5)}$, el número de lactantes de 0 a 6 meses que son alimentados de manera exclusiva con leche materna es bajo, siendo más frecuente la alimentación con fórmulas lácteas, los cuales no sustituye a los componentes nutricionales propios de la leche materna, son sólo un alimento más que se le da al niño ${ }^{(6)}$, que dista de igualar a la leche materna ${ }^{(2)}$. Según la OMS, la mayoría de lactantes reciben otro tipo de alimentos en los primeros meses de vida y, solamente en cantidad mínima reciben lactancia materna exclusiva durante los primeros seis meses de vida.

El consumo de fórmulas como sustituto de la leche materna, otros tipos de líquido y el inicio temprano de la alimentación complementaria, son los determinantes de la desnutrición crónica en los niños menores de dos años ${ }^{(7)}$. Asimismo, las fórmulas lácteas tienen alta publicidad, lo cual influye en las preferencias para adquirir variedad de productos lácteos. La alimentación del lactante con fórmulas lácteas coloca al infante frente a un riesgo mayor de padecer infecciones y enfermedades ${ }^{(8-10)}$ como la obesidad desde la niñez ${ }^{(11-13)}$.

Muchos de los lactantes tienen alteraciones en el estado nutricional, básicamente por el abandono de la lactancia materna exclusiva (14). Además, tal como señala la OMS ${ }^{(15)}$, la morbilidad es muy prevalente en niños que no reciben la lactancia materna exclusiva. También, Hernández y Alcázar ${ }^{(16,17)}$ evidenciaron que la mayoría de los niños padecían de anemia moderada por interrupción de la lactancia materna exclusiva. En este contexto, también es importante y decisivo en el desarrollo del niño el nivel de hemoglobina para detectar oportunamente la presencia de la anemia ferropénica ${ }^{(18)}$. En un estudio desarrollado por Fernández y Mamani ${ }^{(19)}$, de los 80 lactantes de 0 a 6 meses de edad hospitalizados, el $55 \%$ presentaba anemia.

Al respecto, Santamarina et al. ${ }^{(20)}$ hallaron que la mayoría de los niños en este estudio padecían de anemia ligera. La deficiencia de hierro fue la causa más frecuente de anemia en la infancia y esta es favorecida por circunstancias propias de la edad o por factores de riesgo (depósitos escasos pre y posnatales, crecimiento acelerado, infecciones, errores dietéticos y no lactancia materna exclusiva.

En un estudio desarrollado en Cuba en el 2018, Bebert et al. ${ }^{(21)}$ encontraron la existencia de prácticas inadecuadas de lactancia materna exclusiva, provocando morbilidad y mortalidad en los niños. Mientras tanto, en Ecuador, en el 2018, Guatemal (22), halló que los lactantes amamantados tienen seis veces más posibilidades de supervivencia en los primeros meses de vida que los niños no amamantados con lactancia materna exclusiva.

En el Perú, en el 2017, según el Informe de los Indicadores de Resultados de los Programas Presupuestales, 20132018, de la Encuesta Demográfica y de Salud Familiar (ENDES) ${ }^{(23)}$, el 64, $2 \%$ de lactantes menores de seis meses de edad recibieron lactancia materna exclusiva. $Y$ en el 2018, el 66, 4 \% de lactantes menores de seis meses de edad tuvieron lactancia materna exclusiva.

Tal como lo establecen las diversas políticas de salud y la Norma Técnica de Salud para el Control de Crecimiento y Desarrollo de la Niña y el Niño Menor de Cinco Años del Ministerio de Salud ${ }^{(24)}$, es importante realizar el monitoreo del crecimiento y desarrollo de las niñas y niños con la finalidad de mejorar su desarrollo integral a través de la detección precoz de riesgos, alteraciones o trastornos de estos procesos, para su atención oportuna así como para promover prácticas adecuadas de cuidado y crianza a nivel familiar y comunitario. En este contexto, la promoción de la lactancia materna exclusiva exige revisar diversas evidencias científicas para fomentar su adherencia y garantizar su impacto en el estado nutricional del lactante, así como en su nivel de hemoglobina.

Frente a la problemática descrita, es necesario el fortalecimiento de las capacidades en consejería nutricional que brinda el profesional de enfermería, 
responsable de los programas de crecimiento y desarrollo (CRED), cumpliendo un rol importante en la educación para la salud en el contexto de la atención primaria de la salud, mediante actividades preventivo-promocionales, para garantizar un adecuado crecimiento y desarrollo, a fin evitar consecuencias acumulativas y prolongadas. Por tal motivo, el objetivo del estudio fue determinar la relación entre el estado nutricional y el nivel de hemoglobina; el tipo de lactancia y el estado nutricional; el tipo de lactancia y el nivel de hemoglobina del lactante de 6 meses que acudieron al control CRED del Centro de Salud Perú-Corea, Huánuco, durante el 2019.

\section{MÉTODOS}

\section{Tipo de estudio}

El enfoque fue cuantitativo. Se trató de un estudio de tipo retrospectivo, analítico y transversal. El diseño fue correlacional. El estudio se desarrolló durante el 2020.

\section{Población y muestra}

Se trabajó con una población muestral de 564 historias clínicas de lactantes atendidos en el Centro de Salud Perú-Corea, de Huánuco, Perú. Se incluyeron las historias clínicas de lactantes de 6 meses durante el primer semestre del 2019; se excluyeron a las historias clínicas vacíos o con inconsistencias en cualquiera de las variables estudiadas. Se hizo el control de calidad de datos a cada historia clínica.

\section{Instrumentos de recolección de datos}

La técnica usada fue la documentación y los datos fueron recogidos de las historias clínicas, recogiéndose datos de edad, género, peso, talla, estado nutricional nivel de hemoglobina y tipo de lactancia, correspondientes al último dato registrado.

\section{Análisis de datos}

En el análisis inferencial, se realizó un análisis bivariado a través de una prueba no paramétrica de $X^{2}$ de independencia, tabulado en el SPSS V15.0. Para la

Tabla 1. Descripción del tipo de lactancia de los lactantes de 6 meses que acuden al control CRED. Centro de Salud Perú-Corea, Huánuco, 2019

\begin{tabular}{lcc}
\hline \multirow{2}{*}{ Tipo de lactancia } & \multicolumn{2}{c}{$\mathbf{n = 5 6 4}$} \\
\cline { 2 - 3 } & $\mathbf{f i}$ & \% \\
\hline Lactancia materna exclusiva & 370 & 65,6 \\
Lactancia artificial & 49 & 8,7 \\
Lactancia mixta & 145 & 25,7 \\
\hline
\end{tabular}

significancia estadística se tuvo en cuenta el valor $\mathrm{p}<$ de 0,05 .

\section{Aspectos éticos}

La realización del estudio no requirió la aprobación de un comité de ética por tratarse de un estudio retrospectivo con datos secundarios; sin embargo, para la recolección de la información, se solicitaron los permisos respectivos a la dirección del Centro de Salud Perú-Corea. La información fue manejada de forma confidencial; de este modo, la base de datos solo incluyó como dato de identificación el número de historia clínica y no fueron registrados otros datos como el nombre, la dirección u otros de identificación personal.

\section{RESULTADOS}

Al analizar las características generales de los lactantes de 6 meses en estudio, un 54,1 \% (305) fueron del género masculino. Evaluando el tipo de lactancia de los lactantes en estudio, se evidenció que el 65,6 \% (370) continuaba con lactancia materna exclusiva, frente a un 25,7 \% (145) cuyo tipo de lactancia era mixta (ver tabla 1).

Al valorar el estado nutricional de los lactantes en estudio, se halló que en mayor proporción el diagnóstico del estado nutricional fue normal (ver tabla 2).

Tabla 2. Descripción del estado nutricional de los lactantes de 6 meses que acuden al control CRED. Centro de Salud Perú-Corea, Huánuco, 2019

\begin{tabular}{lcc}
\hline & \multicolumn{2}{c}{$\mathbf{n = 5 6 4}$} \\
\cline { 2 - 3 } Estado nutricional & $\mathbf{f i}$ & $\%$ \\
\hline Peso/Edad & & \\
Normal & 478 & 84,8 \\
Obesidad & 86 & 15,2 \\
Talla/Edad & & \\
Normal & 559 & 99,1 \\
Talla baja & 5 & 0,9 \\
Peso/Talla & & \\
Normal & & 94,9 \\
Desnutrición aguda & 535 & 5,1 \\
General & 559 & \\
Normal & 59 & \\
Alterado & & 9,9 \\
\hline
\end{tabular}


Tabla 3. Nivel de hemoglobina de los lactantes de 6 meses que acuden al control CRED. Centro de Salud Perú-Corea, Huánuco, 2019

\begin{tabular}{lcc}
\hline \multirow{2}{*}{ Nivel de hemoglobina } & \multicolumn{2}{c}{$\mathbf{n = 5 6 4}$} \\
\cline { 2 - 3 } & $\mathbf{f i}$ & $\mathbf{\%}$ \\
\hline Normal & 558 & 98,9 \\
Anemia leve & 3 & 0,5 \\
Anemia moderada & 3 & 0,5 \\
\hline
\end{tabular}

Al analizar el nivel de hemoglobina de los lactantes en estudio, se evidenció que, 98,9 \% (558) presentaban un nivel normal de hemoglobina, frente a una mínima proporción [0,5\% (3)] con anemia leve y moderada, respectivamente (ver tabla 3 ).

Al contrastar la relación a nivel inferencial entre el tipo de alimentación y el estado nutricional de los lactantes de 6 meses en estudio, se halló relación entre la lactancia materna exclusiva mientras que el estado nutricional fue normal $\left[\left(x^{2}=25,55 ; p=0,006\right)\right]$ (ver tabla 4$)$.

Al contrastar la relación entre el tipo de lactancia y el nivel de hemoglobina de los lactantes de 6 meses en estudio, se evidenció relación entre la lactancia materna exclusiva y un nivel normal de hemoglobina $\left[\left(x^{2}=30,71\right.\right.$; $p=0,000)$ ] (ver tabla 5).

Finalmente, al contrastar la relación entre el estado nutricional y el nivel de hemoglobina de los lactantes, se halló relación significativa entre el estado nutricional normal y el nivel normal de hemoglobina $\left[\left(x^{2}=488,13\right.\right.$; $p=0,000)]$ (ver tabla 6).

Tabla 5. Relación entre el tipo de alimentación y el nivel de hemoglobina de los lactantes de 6 meses que acuden al control CRED. Centro de Salud Perú Corea, Huánuco, 2019

\begin{tabular}{|c|c|c|c|c|c|c|c|c|}
\hline \multirow{3}{*}{$\begin{array}{l}\text { Nivel de } \\
\text { hemoglobina }\end{array}$} & \multicolumn{6}{|c|}{ Tipo de lactancia } & \multirow{3}{*}{$X^{2}$} & \multirow{3}{*}{ p-valo } \\
\hline & \multicolumn{2}{|c|}{$\begin{array}{c}\text { Lactancia } \\
\text { materna } \\
\text { exclusiva }\end{array}$} & \multicolumn{2}{|c|}{$\begin{array}{l}\text { Lactancia } \\
\text { artificial }\end{array}$} & \multicolumn{2}{|c|}{$\begin{array}{l}\text { Lactancia } \\
\text { mixta }\end{array}$} & & \\
\hline & fi & $\%$ & fi & $\%$ & fi & $\%$ & & \\
\hline Normal & 432 & 76,6 & 39 & 6,9 & 87 & 15,4 & & \\
\hline Anemia leve & 0 & 0,0 & 0 & 0,0 & 3 & 0,5 & & \\
\hline $\begin{array}{l}\text { Anemia } \\
\text { moderada }\end{array}$ & 0 & 0,0 & 0 & 0,0 & 3 & 0,5 & 30,71 & 0,000 \\
\hline Total & 432 & 76,6 & 39 & 6,9 & 93 & 16,5 & & \\
\hline
\end{tabular}

Tabla 4. Relación entre el tipo de alimentación y el estado nutricional de los lactantes de 6 meses que acuden al control CRED. Centro de Salud Perú-Corea, Huánuco, 2019

\begin{tabular}{|c|c|c|c|c|c|c|c|c|}
\hline \multirow{3}{*}{$\begin{array}{l}\text { Estado } \\
\text { nutricional }\end{array}$} & \multicolumn{6}{|c|}{ Tipo de lactancia } & \multirow{3}{*}{$\mathrm{X}^{2}$} & \multirow{3}{*}{ p-valor } \\
\hline & \multicolumn{2}{|c|}{$\begin{array}{l}\text { Lactancia } \\
\text { materna } \\
\text { exclusiva }\end{array}$} & \multicolumn{2}{|c|}{$\begin{array}{l}\text { Lactancia } \\
\text { artificial }\end{array}$} & \multicolumn{2}{|c|}{$\begin{array}{l}\text { Lactancia } \\
\text { mixta }\end{array}$} & & \\
\hline & fi & $\%$ & fi & $\%$ & fi & $\%$ & & \\
\hline Normal & 432 & 76,6 & 39 & 6,9 & 88 & 15,6 & & \\
\hline Alterado & 0 & 0,0 & 0 & 0,0 & 5 & 0,9 & 25,55 & 0,000 \\
\hline Total & 432 & 76,6 & 39 & 6,9 & 93 & 16,5 & & \\
\hline
\end{tabular}

\section{DISCUSIÓN}

En un primer momento se analizó la relación entre el tipo de alimentación con lactancia materna exclusiva y el estado nutricional normal de los lactantes de 6 meses en estudio; nuestro resultado es coincidente con el estudio de Guatemal ${ }^{(22)}$, quien halló que la lactancia materna exclusiva está relacionada con el estado nutricional.

Choque ${ }^{(5)}$ halló que existe relación directa entre los tipos de lactancia y el estado nutricional de los lactantes. También Ubillús et al. ${ }^{(25)}$ hallaron un $92,8 \%$ de lactantes con diagnóstico nutricional normal o eutrófico, quienes fueron alimentados con lactancia materna exclusiva. Argote y Cordero $^{(7)}$ encontraron que los niños que reciben lactancia materna exclusiva tienen un estado nutricional normal. También, Rodríguez, et al. ${ }^{(26)}$ encontraron que la lactancia materna es un protector de la malnutrición por exceso (sobrepeso, obesidad), independiente del peso al nacer. Y, Sosa et al. ${ }^{(27)}$ hallaron una evidente primacía

Tabla 6. Relación entre el estado nutricional y el nivel de hemoglobina de los lactantes de 6 meses que acuden al control CRED. Centro de Salud Perú-Corea, Huánuco, 2019

\begin{tabular}{|c|c|c|c|c|c|c|}
\hline \multirow{3}{*}{$\begin{array}{l}\text { Nivel de } \\
\text { hemoglobina }\end{array}$} & \multicolumn{4}{|c|}{ Estado nutricional } & \multirow{3}{*}{$X^{2}$} & \multirow{3}{*}{ p-valor } \\
\hline & \multicolumn{2}{|c|}{ Normal } & \multicolumn{2}{|c|}{ Alterado } & & \\
\hline & fi & $\%$ & fi & $\%$ & & \\
\hline Normal & 558 & 98,9 & 0 & 0,0 & & \\
\hline Anemia leve & 1 & 0,2 & 2 & 0,4 & & \\
\hline $\begin{array}{l}\text { Anemia } \\
\text { moderado }\end{array}$ & 0 & 0,0 & 3 & 0,5 & 488,13 & 0,000 \\
\hline Total & 559 & 99,1 & 5 & 0,9 & & \\
\hline
\end{tabular}


de niños y niñas con estado nutricional normal entre los alimentados con leche materna exclusiva y los que nacieron con peso adecuado para la edad. El estudio que contradice nuestros resultados es el de Mosqueira (28), quien concluyó que no existe asociación significativa entre el tipo de lactancia y la presencia de alguna forma de desnutrición, pero la lactancia materna exclusiva favorece en un mayor porcentaje el estado nutricional normal que los otros tipos de lactancia.

En un segundo momento, respecto a que el tipo de lactancia materna exclusiva y el nivel normal de hemoglobina de los lactantes de 6 meses en estudio tienen relación, nuestros resultados concuerdan con el estudio de Puescas y Chapilliquen ${ }^{(29)}$, quienes hallaron que la lactancia materna exclusiva es un factor protector $(O R=0,018)$; es decir, un niño que recibe lactancia exclusiva tiene 0,018 veces de posibilidad de presentar anemia que un niño que recibe lactancia mixta. También, Cóndor y Baldeón ${ }^{\left({ }^{30}\right)}$ concluyeron que los factores relacionados con el desarrollo de la anemia fueron la lactancia materna no exclusiva, la inadecuada suplementación con multimicronutrientes y la presencia de enfermedad diarreica aguda, los cuales resultaron significativos con relación a la presencia de anemia en los niños de 6 a 36 meses.

Sin embargo, Marques et al. ${ }^{(31)}$ en un estudio realizado en Brasil, demostraron que la lactancia materna exclusiva fue un factor protector de anemia solo hasta los 4 meses y que, posterior a ese tiempo, incrementaba su riesgo. En tanto Pasricha et al. ${ }^{(32)}$, encontraron que la anemia estaba directamente asociada a la continuidad de la lactancia materna más de 6 meses. Resultados similares obtuvieron Santamarina et al. ${ }^{(20)}$, en niños cubanos de 0 a 6 meses, donde el $100 \%$ había recibido lactancia materna y, sin embargo, el 71,43\% tenía anemia ligera y el 28, $57 \%$ anemia moderada. Mientras que Zelaya et al. ${ }^{(33)}$ hallaron que los lactantes que recibieron lactancia mixta y materna exclusiva no presentaron una diferencia evidente en sus niveles de hemoglobina.

Respecto a las limitaciones del estudio se reconoce que, al ser un estudio retrospectivo, de una fuente secundaria no se tienen datos como peso al nacer, patología al nacer, frecuencia de enfermedades infecciosas, entre otras, así como variables de la madre relacionadas al tipo de parto, o $\mathrm{Hb}$ prenatal, que permitirían realizar estudios multivariados.

En un tercer momento, se halló relación entre el estado nutricional normal y el nivel de hemoglobina normal; estos resultados coinciden con el estudio de
Pérez et al. ${ }^{(34)}$, quienes encontraron que el $81,13 \%$ de niños con peso normal para la talla presentaron valores normales en sus niveles de hemoglobina.

También, Gómez y Munares ${ }^{(35)}$ lograron determinar la presencia de anemia y desnutrición crónica en los lactantes y con diferencias según edad, altitud, regiones de residencia y tipo de establecimiento de salud; $y$ en desnutrición crónica incluso según sexo. Además, indicaron que a mayor probabilidad de anemia en desnutridos crónicos.

Nuestro resultado se contradice, sin embargo, con el estudio de Mendoza (36), quien encontró, mediante la ecuación estadística de coeficiente de correlación de Pearson, que no existía relación significativa entre las variables estudiadas, estado nutricional, nivel de hemoglobina y consumo de hierro.

Farfán ${ }^{(37)}$, también halló que no existe relación entre el estado nutricional y la anemia ferropénica; teniendo un buen estado nutricional existen diversos factores que ocasionan la anemia en los niños comprendidos entre las edades estudiadas, así como que los niños que presentaban desnutrición o sobrepeso pueden o no presentar anemia ferropénica.

Finalmente, se concluye que existe relación entre el estado nutricional normal y el nivel normal de hemoglobina, la lactancia materna exclusiva y un nivel normal de hemoglobina; asimismo, se halló relación entre la lactancia materna exclusiva y el estado nutricional normal de los lactantes. Sin embargo, estos hallazgos requieren realizar mayores estudios.

Por todo lo anterior, se recomienda que el personal de salud debe garantizar el cumplimiento de las disposiciones técnicas para la evaluación oportuna y periódica del crecimiento y desarrollo del niño menor de 5 años; en ese sentido, una función fundamental que se realiza durante la atención de CRED es la educación y evaluación de la técnica correcta del amamantamiento para favorecer la lactancia efectiva.

También, es necesario realizar un buen registro de la historia clínica, enfatizando el estado nutricional y el tipo de lactancia que recibe el lactante de 6 meses; asimismo se pueden considerar variables adicionales, ahondado más sobre la alimentación que recibe el niño menor de 6 meses. Además, es necesario contar con un plan de atención de salud individualizado del niño y políticas de protección de la práctica de la lactancia materna exclusiva de manera articulada. 
Por último, es necesario seguir desarrollando investigaciones prospectivas y comparativas en el contexto académico, donde se evalúa la anemia según mes de edad y debido a que diversos estudios señalan que la lactancia materna exclusiva solo protege a los lactantes menores de 4 meses.

\section{REFERENCIAS}

1. Jiménez R, Curbelo JL, Peñalver R. Relación del tipo de alimentación con algunas variables del crecimiento, estado nutricional y morbilidad del lactante. Colombia Méd [Internet] 2005; 36(4, Supl 3): 19-25 [Consultado 2020 Dic 15] Disponible en: https://colombiamedica. univalle.edu.co/index.php/comedica/article/view/395

2. Barriuso L, Miguel MD, Sánchez M. Lactancia materna: factor de salud. Recuerdo histórico. Anales Sis San Navarra [Internet] 2007; 30(3): 383-91 [Consultado 2020 Dic 15] Disponible en: http://scielo.isciii.es/scielo.php?scrip$\mathrm{t}=\mathrm{sci}$ _arttext\&pid=S1137-66272007000500007

3. Valenzuela-Duarte AD, Soto-Torres ME, García-Pastoressa $M$, et al. Capacitación del personal de enfermería en lactancia materna. Rev Enferm IMSS [Internet] 2008; 16(2): 109-114 [Consultado 2020 Dic 12] Disponible en: https://www.medigraphic.com/pdfs/enfermeriaimss/ eim-2008/eim082i.pdf

4. Constantino $M H$, Struchiner CJ, Serrano MT, Kac G. Effect of predominant breastfeeding duration on infant growth: prospective study using nonlinear mixed effect models. J. Pediatr. (Rio J.) [Internet] 2008 Jun; 84(3): 237-243 [Consultado 2020 Dic 12] Disponible en: http://www.scielo.br/scielo.php?script=sci_arttext\&pi$\mathrm{d}=$ S0021-75572008000300009\&lng=en. https://doi. org/10.2223/JPED.1795

5. Choque Flores MDC. Tipos de lactancia y estado nutricional en lactantes de 6 meses en el Centro de Salud Metropolitano de Puno - 2014 [Internet] Puno: Universidad Nacional del Altiplano. Facultad de Enfermería. Escuela Profesional de Enfermería; 2015 [Consultado 2020 Dic 14] Disponible en: http://repositorio.unap.edu.pe/handle/UNAP/1939

6. Lactancia materna [Internet] Fondo de las Naciones Unidas para la Infancia (UNICEF); 2015 [Consultado 2019 Jul 16] Disponible en: https://www.unicef.org/spanish/ nutrition/index_24824.html

7. Argote $\mathrm{D}$, Cordero J. Relación del tipo de lactancia y el estado, nutricional de los niños menores de seis" I meses-CS Chilca 2014 [Internet] Huancayo: Universidad Nacional del Centro del Perú; 2015 [Consultado 2020 Dic 14] Disponible en: http://repositorio.uncp.edu.pe/handle/UNCP/1068

8. Bartolomé L. Relación entre el nivel de conocimiento de la madre sobre alimentación complementaria y estado nutricional del niño de 6 a 12 meses de edad, que asiste al consultorio de crecimiento y desarrollo del centro de salud Supte San Jorge, Tingo Maria-2016 [Internet] Huánuco: Universidad Nacional Hermilio Valdizan; 2017 [Consultado 2020 Dic 10] Disponible en: https:// alicia.concytec.gob.pe/vufind/Record/UDHR_76ceb7e261355bd46ccdd2cdb24b4373

9. Amaya N. Prácticas alimentarias de la madre y estado nutricional del lactante de 6 a 12 meses. Puesto de salud garatea. Nuevo chimbote-2014 [Internet] Chimbote:
Universidad Nacional del Santa; 2017 [Consultado 2020 Dic 15] Disponible en: http://repositorio.uns.edu.pe/ bitstream/handle/UNS/2877/42879.pdf?s

10. Nutrición humana en el mundo en desarrollo [Internet] Organización de las Naciones Unidas para la Alimentación y la Agricultura; 2020 [Consultado 2016 Oct 31] Disponible en: http://www.fao.org/docrep/006/w0073s/ w0073s0c.htm\#TopOfPage

11. Alimentación del lactante y del niño pequeño [Internet] Organización Mundial de la Salud (OMS); 2020 [Consultado 2016 Ago 20] Disponible en: http://www.who.int/ mediacentre/factsheets/fs342/es/

12. Jiménez $C$. Inicio de la alimentación complementaria y estado nutricional en lactantes de 6 a 12 meses que acuden al Centro de Salud Fortaleza, 2008 [Internet] Lima: Universidad Nacional Mayor de San Marcos. Facultad de Medicina; 2008 [Consultado 2020 Dic 12] Disponible en: https://cybertesis.unmsm.edu.pe/handle/20.500.12672/544

13. Perú. Ministerio de Salud. La Lactancia Materna y el Cumplimiento del Código Intencional de Comercialización de sucedáneos de la Leche Materna en el Perú [Internet] [Consultado 2017 Nov 20] Disponible en: http:// bvs.minsa.gob.pe/local/minsa/1710.pdf

14. Brahm P, Valdés V. Beneficios de la lactancia materna y riesgos de no amamantar. Rev. chil. pediatr. [Internet] 2017; 88(1): 07-14 [Consultado 2020 Dic 17] Disponible en: https://scielo.conicyt.cl/scielo.php?script=sci_arttext\&pid=S0370-41062017000100001\&lng=es.

15. Lactancia Materna Exclusiva [Internet] Organización Mundial de la Salud (OMS); 2019 [Consultado 2019 Jul 16] Disponible en: https://www.who.int/nutrition/topics/exclusive_breastfeeding/es/.

16. Hernández M. Alimentación complementaria. Curso de actualización. Ped [Internet] 2006; 3(1): 12-43 [Consultado 2020 Dic 14] Disponible en: https://www.aepap.org/ sites/default/files/complementaria.pdf

17. Alcázar Zambrano J. Anemia y embarazo [Internet] Pamplona: Clínica Universidad de Navarra; 2011 [Consultado 2019 Jul 16] Disponible en: http://www.cun.es/area-salud/perfil/futura-mama/anemia-embarazo

18. Laguna L. Nivel de hemoglobina del nacimiento y estado nutricional posterior de lactantes de 0 a 6 meses de madres adolescentes usuarias del centro de salud de la micro red Amarilis, Huánuco 2016 [Internet] Huánuco: Universidad Nacional Hermilio Valdizán; 2016 [Consultado 2020 Dic 15] Disponible en: https://alicia.concytec. gob.pe/vufind/Record/UNHE_586928597982aee41f36b5a9fa355629

19. Fernández-Oliva JD, Mamani-Urrutia VA. Niveles de hemoglobina en lactantes de 0 a 6 meses de edad hospitalizados en el Instituto Nacional de Salud del Niño, 2015. An. Fac. med. [Internet] 2019 Ene; 80(1): 45-50 [Consultado 2020 Nov 28] Disponible en: http:// www.scielo.org.pe/scielo.php?script=sci_arttext\&pi$\mathrm{d}=$ S1025-55832019000100008\&lng=es.

20. Santamarina A, Sánchez RD, Alba O. Caracterización de lactantes menores de 6 meses con anemia ferropénica. Rev Cubana Pediatr [Internet] 2017; 89(1): 11-19 [Consultado 2020 Nov 17] Disponible en: https://www. medigraphic.com/cgi-bin/new/resumen.cgi?IDARTICU$\mathrm{LO}=72239$ 
21. Bebert $Y$, Medina $M$, Torres IE, Ramírez L, Borot Y. Lactancia materna y efecto del destete precoz en el estado nutricional y morbilidad de los lactantes en municipio Ribas, Venezuela. Rev Inf Científica [Internet] 2018; 97(2): 315-23 [Consultado 2020 Dic 17] Disponible en: https:// www.medigraphic.com/cgi-bin/new/resumen.cgi?lDARTICULO=81194

22. Guatemal J. Estado nutricional y prácticas de lactancia materna en niños menores de un año de la población de Tanguarín, San Antonio de Ibarra en el periodo 20162017 [Internet] Ibarra: Universidad Técnica del Norte; 2018 [Consultado 2020 Dic 17] Disponible en: http:// repositorio.utn.edu.ec/handle/123456789/8420

23. Instituto Nacional de Estadística e Informática (INEI). Indicadores de resultados de los programas presupuestales, 2013 - 2018. Perú [Internet] [Consultado 2019 Jul 16] Disponible en: https://proyectos.inei.gob.pe/endes/2018/ppr_2013_2018/Indicadores\%20de\%20Resultados\%20de\%20los\%20Programas\%20Presupuestales_ENDES_2018.pdf.

24. Perú. Ministerio de Salud. Norma Técnica de Salud para el Control de Crecimiento y Desarrollo de la Niña y el Niño Menor de Cinco Años. Resolución Ministerial N. ${ }^{\circ}$ 537-2017-MINSA (Jul 10 2017). Disponible en: http:// www.redsaludcce.gob.pe/Modernidad/archivos/dais/ ppan/normast/CRED.pdf

25. Ubillús G, Lamas C, Lescano B, Lévano M, Llerena Z, López, $R$, Estado nutricional del lactante de uno a seis meses y tipo de lactancia. Horizonte Médico [Internet] 2011; 11(1): 19-23 [Consultado 2020 Dic 22] Disponible en: https://www.redalyc.org/articulo.oa?id=371637121003

26. Rodríguez N, Fernández-Britto JE, Martínez TP, Martínez $\mathrm{R}$, Castañeda CM, Chediak Ml, et al. Asociación de la lactancia materna y el estado nutricional en niños de 7-11 años con alto peso al nacer. Rev Cubana Invest Bioméd [Internet] 2018 Sep; 37(3): 1-14 [Consultado 2020 Dic 4] Disponible en: http://scielo.sld.cu/scielo.php?script=sci_arttext\&pid=S0864-03002018000300009\&lng=es

27. Sosa M, Ramírez MC, Suárez D, Lohuan S, Gómez I. Evaluación nutricional de lactantes menores de un año en el área de salud "Josué País García". MEDISAN [Internet] 2011 Jul; 15(7): 952-957 [Consultado 2020 Dic 15] Disponible en: http://scielo.sld.cu/scielo.php?script=sci_arttext\&pid=S1029-30192011000700010\&lng=es

28. Mosqueira KJ. Estado nutricional de lactante de uno a seis meses en relación al tipo lactancia en Centro de Salud Progreso 2016 [Internet] Chimbote: Universidad San Pedro Facultad Medicina Humana Escuela de Medicina; 2017 [Consultado 2020 Dic 13] Disponible en: http://repositorio.usanpedro.edu.pe/handle/USANPEDRO/809

29. Puescas VF, Chapilliquen V. Factores asociados a la anemia en niños de 6 a 36 meses de edad atendidos en el centro médico Leoncio Amaya Tume Essalud - La Unión, primer semestre 2019 [Internet] Piura: Universidad Na- cional De Piura. Facultad de Ciencias. Escuela Profesional de Estadística; 2019 [Consultado 2020 Dic 13] Disponible en: http://repositorio.unp.edu.pe/handle/UNP/2031

30. Cóndor-Cisneros J, Baldeón-Wong E. Anemia en niños de 6 a 36 meses en un Centro de Salud urbano. Huánuco, 2016. Rev Peru Investig Salud [Internet] 2019; 3(3): 109115 [Consultado 2020 Dic 3] Disponible en: http://revistas.unheval.edu.pe/index.php/repis/article/view/332

31. Marques RFSV, Taddei JAAC, López FA, Braga JAP, Marques RFSV, Taddei JAAC, etal. Breastfeeding exclusively and iron deficiency anemia during the first 6 months of age. Rev Assoc Médica Bras. 2014; 60(1): 18-22.

32. Pasricha R, Black J, Muthayya S, Shet A, Bhat V, Nagaraj $S$, et al. Determinants of anemia among young children in rural India. Pediatrics [Internet] 2010; 126(1): e140-9 [Consultado 2020 Dic 3] doi: https://doi.org/10.1542/ peds.2009-3108. https://pubmed.ncbi.nlm.nih. gov/20547647/

33. Zelaya-Lozano E, Peña-Hernández A, París-Domínguez I, Madrid-Fígueroa J. Anemias y reservas de hierro en niños menores de 24 meses aparentemente sanos. Honduras Pediátrica [Internet] 1997; 18(2): 38-41[Consultado 2020 Nov 23] Disponible en: http://www.bvs.hn/ $\mathrm{RHP} / \mathrm{pdf} / 1997 / \mathrm{pdf} /$ Vol18-2-1997-4.pdf

34. Pérez-Avila YF, Pérez-González OF, Yabor-Palomo AM, Laborí-Quesada P, Benítez-Rojas L. Estado nutricional y niveles de hemoglobina en niños menores de cinco años en el área de salud del policlínico "Gustavo Aldereguía Lima". Rev. electron. Zoilo [Internet] 2019; 44(4): [aprox. 0 p.] [Consultado 2020 Nov 27] Disponible en: http://revzoilomarinello.sld.cu/index.php/zmv/article/ view/1870

35. Gómez-Guizado G, Munares-García O. Anemia y estado nutricional en lactantes de dos a cinco meses atendidos en establecimientos del Ministerio de Salud del Perú, 2012. Revista Peruana de Medicina Experimental y Salud Pública [Internet] 2014; 31(3): 487-493 [Consultado 2020 Dic 3] Disponible en: https://rpmesp.ins.gob.pe/ index.php/rpmesp/article/view/85/1924

36. Mendoza YL. Estado nutricional, nivel de hemoglobina y consumo de hierro en niños de 6 a 59 meses de edad de los establecimientos de salud de los distritos de Puno y Azángaro 2018 [Internet] Puno: Universidad Nacional del Altiplano; 2018 [Consultado 2020 Dic 13] Disponible en: http://repositorio.unap.edu.pe/bitstream/handle/ UNAP/9955/Mendoza_Larico_Yudy_Lizeth.pdf?sequence $=1$ \&isAllowed $=y$

37. Farfán C. Relación del estado nutricional y anemia ferropénica en niños menores de dos años evaluados en el Centro de Salud Materno infantil Miguel Grau 2012. [Internet] Lima: Universidad Peruana Unión; 2015 [Consultado 2020 Dic 21] Disponible en: https://repositorio. upeu.edu.pe/bitstream/handle/UPEU/141/Catalina_Tesis_bachiller_2015.pdf?sequence $=1$ \&isAllowed $=y$ 